\title{
Scientific Note/Comunicacão Cientifica Registro de Tetranychus mexicanus (McGregor) (Acari: Tetranychidae) em mamoeiro (Carica papaya L.) no estado do Acre, Brasil
}

\author{
Rodrigo Souza Santos ${ }^{1 凶}$, Noeli Juarez Ferla², Júlia Jantsch Ferla² \& Wangerlândia da Silva ${ }^{1}$
}

1. Embrapa Acre. 2.Universidade do Vale do Taquari - UNIVATES.

\section{EntomoBrasilis 11 (2): 147-150 (2018)}

Resumo. Foi efetuado o primeiro registro do ácaro Tetranychus mexicanus (McGregor) em mamoeiro no Município de Rio Branco, Acre. Foi detectada a presença de teia, perda de área fotossintetizante, necrose e secamento das folhas das plantas infestadas. Este é o primeiro registro dessa espécie de ácaro no estado do Acre.

Palavras-chave: Ácaro fitófago; Amazônia; Caricaceae; fruticultura; Tetranychoidea.

\section{Record of Tetranychus mexicanus (McGregor) (Acari: Tetranychidae) in papaya plant (Carica} papaya L.) in the Acre State, Brazil

\begin{abstract}
The occurrence of the spider mite Tetranychus mexicanus (McGregor) in papaya plants was reported in the municipality of Rio Branco, Acre state, Brazil. The leaves of the infested plants presented web, loss of photosynthesizing area, necrosis and drying. This is the first record of this species of mite in the Acre State.
\end{abstract}

Keywords: Amazon; Caricaceae; fruticulture; Phytophagous mite; Tetranychoidea.

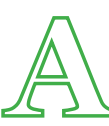
cultura do mamoeiro, Carica papaya L. (Caricaceae), conhecido popularmente como "mamão papaia", exerce uma grande importância socioeconômica para a fruticultura nacional, destacando o Brasil como segundo produtor mundial e o terceiro exportador da fruta, com área plantada de cerca de 30 mil ha, uma produção de mais de 1,4 milhão de ton. e produtividade média de aproximadamente 47 mil Kg/ha (FAO 2016). O mamão é cultivado em quase todo o território brasileiro, sendo os estados da Bahia, Espírito Santo e Ceará, os principais responsáveis pela maior parte da produção nacional (FARIA et al. 2009). No ano de 2016, 323 ha com a cultura do mamoeiro haviam sido plantados no estado do Acre, principalmente por pequenos produtores de base familiar, nos municípios de Acrelândia, Porto Acre, Sena Madureira e Senador Guiomard (IBGE 2016).

O fruto possui elevado valor nutricional, sendo rico em açúcares e compostos bioativos (carotenoides e vitamina C) e tem sabor e aroma agradáveis, pela presença de compostos voláteis. Quando verde, o mamão possui elevados teores da enzima papaína, empregada nas indústrias alimentícia, farmacêutica e de cosméticos. Desta planta também é extraído o alcaloide carpaína, um ativador cardíaco (DANTAS et al. 2013).
Concomitantemente ao crescimento da importância do cultivo do mamoeiro, aliada à expansão da área plantada em regime de monocultura, os problemas fitossanitários têm se agravado, principalmente no que tange ao ataque de insetos e ácaros (GALLO et al. 2002). No mundo, já foram registradas aproximadamente 209 espécies de artrópodes pertencentes a oito ordens e sete famílias, associadas ao mamoeiro (Culik et al. 2003). Nesse inventário, 83 espécies (39,7\%) ocorrem no Brasil e 50 espécies já foram registradas em mamoeiro (Culik et al. 2003). Dentre os artrópodes associados ao mamoeiro no Brasil, os ácaros são considerados pragas-chave dessa cultura, destacando-se os ácaros: branco, Polyphagotarsonemus latus (Banks) (Acari: Tarsonemidae) e rajado, Tetranychus urticae (Koch) (Acari: Tetranychidae) (MARTINS \& MARIN 1998).

Em agosto de 2017 foi observada uma infestação de ácaros em plantas de mamoeiro em dois locais distintos no Município de Rio Branco, AC, sendo: local 1 (o9 $\left.{ }^{\circ} 56^{\prime} 40,5^{\prime \prime} \mathrm{S} ; 67^{\circ} 57^{\prime} 47,4^{\prime \prime} \mathrm{O}\right)$ e local 2 (09 ${ }^{\circ} 58^{\prime} 30,6$ " S; 67 48'28,3” O). As plantas observadas eram individuais e os locais não eram áreas comerciais de cultivo do mamoeiro no Estado.

Foi coletada uma amostra de folhas infestadas (duas folhas por planta) em cada localidade, as quais foram acondicionadas em sacos plásticos identificados e levadas até o Laboratório de
Edited by:

William Costa Rodrigues

\section{Article History:}

Received: 08.ii.2018

Accepted: 07.iv.2018
Corresponding author:

Rodrigo Souza Santos

७ rodrigo.s.santos@embrapa.br

(3) http://orcid.org/0000-0002-0879-0049
Funding agencies:

$\Delta$ Without funding declared 
Entomologia da Embrapa Acre. Em laboratório, o material foi triado e os ácaros foram preservados em frasco de vidro contendo álcool a 70\% e enviados para identificação. Os ácaros foram analisados (36 ㅇ e 4 §̂), montados em lâminas permanentes com meio de Hoyer e identificados em nível específico com auxílio de chave de identificação de BAKER \& TutTle (1994). As lâminas foram depositadas na Coleção de referência do Museu de Ciências Naturais da Universidade do Vale do Taquari (ZAUMCN) - UNIVATES, Lajeado, RS.

Os espécimes foram identificados pelo coautor do trabalho, como Tetranychus mexicanus (McGregor) (Acari: Tetranychidae), sendo as estrias pré-genitais, quetotaxia do tarso I das fêmeas e o edeago dos machos, características diagnósticas da espécie (Figuras 1 A, B e C). A coloração das fêmeas dessa espécie varia do verde ao vermelho e os machos possuem tonalidades que variam do verde-amarelado ao vermelho (FERES 2000). Foi observado que as colônias de T. mexicanus localizavamse preferencialmente na face abaxial das folhas e, em menor quantidade na face adaxial. As folhas infestadas apresentavam perda de área fotossintetizante (áreas cloróticas), progredindo para necrose foliar, secamento e morte (Figuras 2 A e B).

A família Tetranychidae abriga uma quantidade relativamente grande de espécies de ácaros estritamente fitógagos, os quais são referidos na literatura nacional como "ácaros-de-teia", devido ao comportamento de muitas espécies de produzirem uma quantidade variável de teia, as quais utilizam para se deslocarem entre plantas (Moraes \& Flechtmann 2008). Os tetraniquídeos são comumente polífagos e algumas espécies possuem especificidade por plantas hospedeiras (GALlo et al. 2002; Moraes \& Flechtmann 2008).

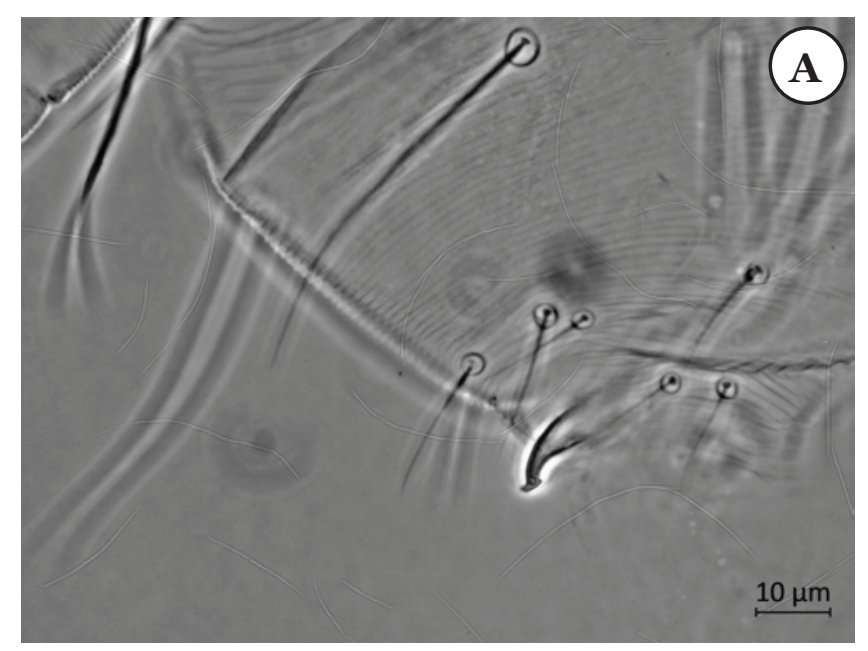

A espécie T. mexicanus já foi registrada em 13 países, todos do Continente Americano, e tendo como hospedeiras 90 espécies vegetais (Bolland et al. 1998), sendo seus primeiros relatos no México, Estados Unidos (Texas) e Argentina, tendo sempre plantas cítricas como hospedeiras (FLECHTMANN \& BAKER 1970).

No Brasil, T. mexicanus já foi registrado em associação com plantas das seguintes famílias: Passifloraceae: maracujazeiro (Passiflora spp.); Malvaceae: cacaueiro (Theobroma cacao L.) e algodoeiro (Gossypium herbaceum L.); Euphorbiaceae: seringueira (Hevea spp.) e mamona (Ricinus communis L.); Arecaceae: coqueiro (Cocos nucifera L.) e pupunheira (Bactris gasipaes Kunth.); Fabaceae: feijoeiro (Phaseolus vulgaris L.) e árvore-de-coral (Erythrina indica Lam.); Musaceae: bananeira (Musa spp.); Rutaceae: citros (Citrus spp.); Caricaceae: mamoeiro (C. papaya); Rosaceae: pereira (Pyrus communis L.) e morangueiro (Fragaria spp.); Meliaceae: cedrela (Cedrela fissilis Vell.); Phytolaccaceae: guiné (Petiveria sp.); Araceae: filodendro(Philodendron sp.); Orchidaceae: orquídeas; Vitaceae: uveira (Vitis vinifera L.); Annonaceae: gravioleira (Annona muricata L.) e em várias outras frutíferas (FLECHTMANN 1967; Paschoal 1968; Paschoal \& Reis 1968; Paschoal 1970; Nakayama et al. 1987; OliveIra 1987; Bolland et al. 1998; Feres 2000; Gioria 2002; Stein \& Daólio 2004; Argolo 2008; Moraes \& Flechtmann 2008; FARIA et al. 2009; SOUSA et al. 2010; MENDONÇA et al. 2011; ANDRADE-BERTOLO et al. 2013).

Outras espécies deácaros tetraniquídeos: T.urticae, Tetranychus bastosi Tuttle, Baker e Sales, Tetranychus desertorum Banks e Tetranychus neocaledonicus André, já haviam sido registrados, associados ao mamoeiro no Brasil (MORAES \& FLECHTMANN 2008). No entanto, não havia registro de tetraniquídeos infestando o mamoeiro no estado do Acre.

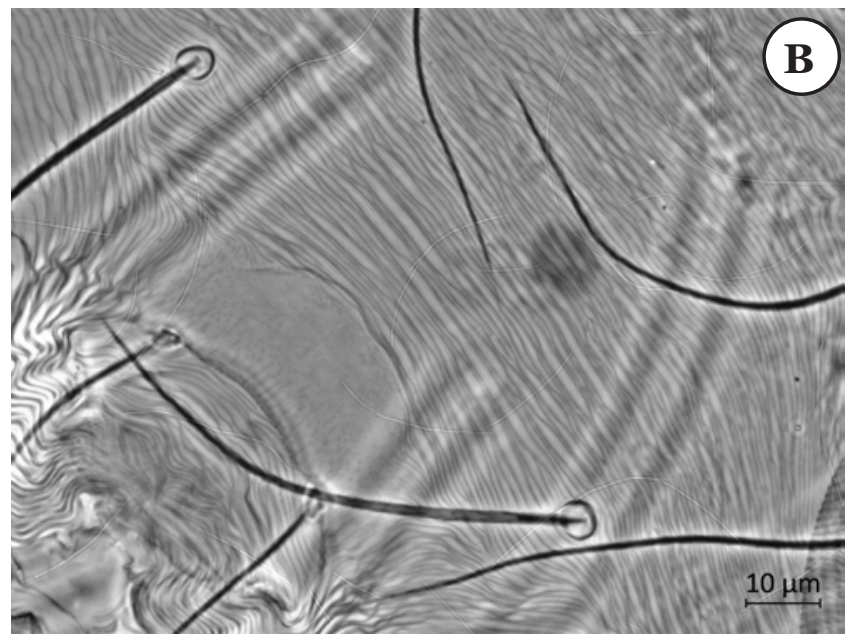

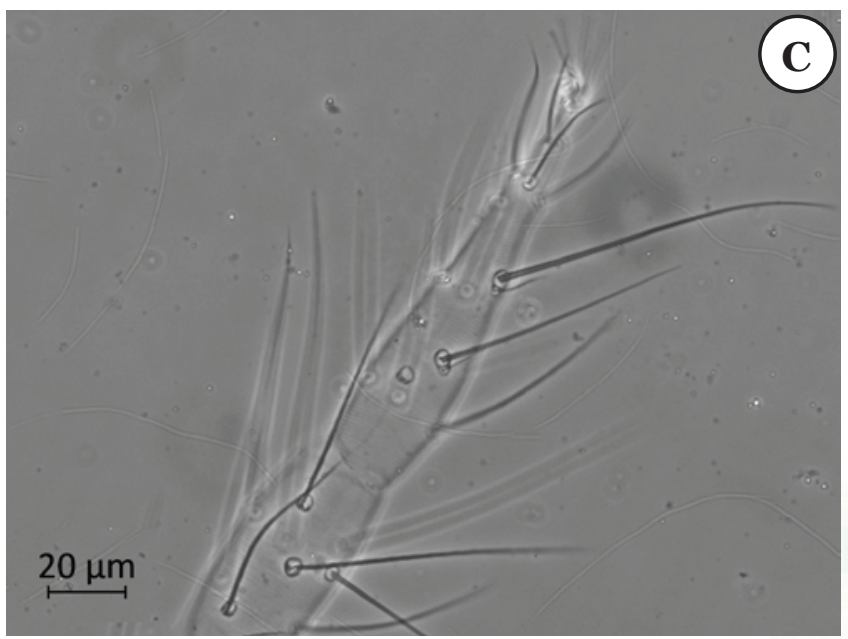

Figura 1. Edeago de macho de Tetranychus mexicanus em vista lateral (A). Estrias pré-genitais fêmea de T. mexicanus (B). Quetotaxia do tarso I de fêmea de T. mexicanus (C). 

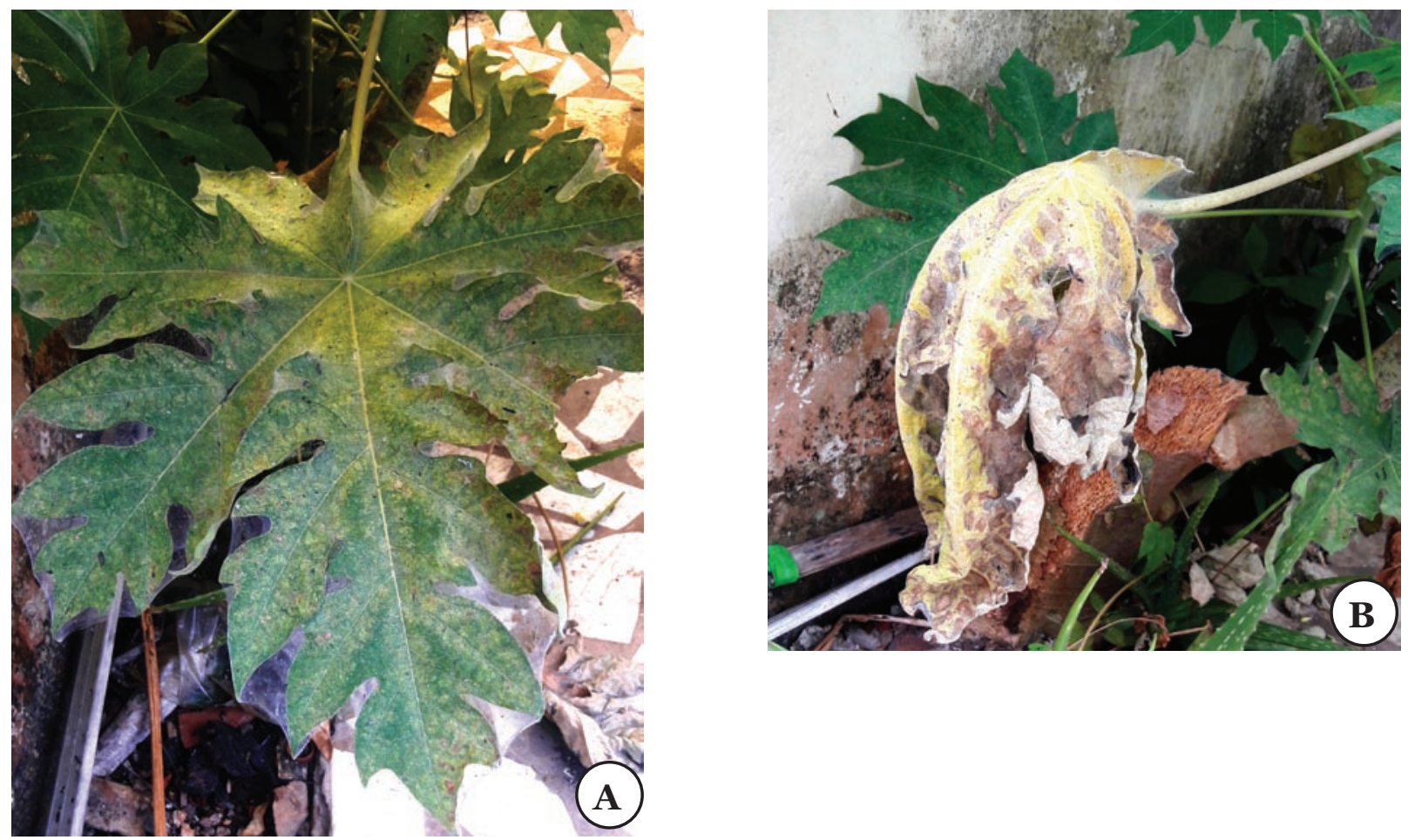

Figura 2. Presença de teia produzida por T. mexicanus em folha de mamoeiro (A). Folha de mamoeiro necrosada e morta pelo ataque de T. mexicanus (B).

Com relação à distribuição geográfica de T. mexicanus, a espécie já havia sido registrada nos estado da Bahia, Minas Gerais, Pernambuco, Paraíba, Rio Grande do Sul e São Paulo (Flechtmann \& Baker 1970; Paschoal 1970; Moraes 1981; Mendonça et al. 2011; ANDRADE-BERTOLO et al. 2013).

Atualmente não há produtos registrados no Ministério da Agricultura, Pecuária e Abastecimento (MAPA) para o controle de T. mexicanus na cultura do mamoeiro no Brasil (AGROFIT 2018), restringindo as opções de controle desse ácaro nessa cultura.

Este relato configura o primeiro registro de ocorrência de $T$. mexicanus no estado do Acre. Como se trata de uma espécie de hábito polífago e que traz severos danos às plantas de mamoeiro infestadas, um monitoramento sistemático deve ser realizado nas áreas produtoras. Ademais, pesquisas no que tange aos métodos de controle são necessárias, a fim de ampliar as formas de combate a este tetraniquídeo.

\section{REFERÊNCIAS}

AGROFIT, 2018. Sistema de Agrotóxicos Fitossanitários do Ministério da Agricultura, Pecuária e Abastecimento (MAPA). Disponível em: <http://agrofit.agricultura.gov.br/agrofit cons/principal agrofit cons $>$. [Acesso em: 08.viii.2018].

Andrade-Bertolo, F. de O., R.B. Moura, A.L. Matioli \& A.P. Ott, 2013. New records of mites (Acari: Prostigmata) on Vitis sp. (L.) in Brazil. Comunicata Scientiae, 4: 414-418.

Argolo, P.S., 2008. Resistência induzida ao ácaro rajado Tetranychus urticae Koch (Acari: Tetranychidae) em morangueiro. Dissertação (Mestrado em Entomologia) Universidade Federal de Viçosa. $53 \mathrm{f}$.

Baker, E.W. \& D.M. Tuttle, 1994. A guide to the spider mites (Tetranychidae) of the United States. Bophal, Indira Publishing House, $347 \mathrm{p}$.

Bolland, H.R., J. Gutierrez \& C.H.W. Flechtmann, 1998. World catalogue of the spider mite family (Acari: Tetranychidae). Leiden, Brill, 392 p.

Culik, M.P., D. dos S. Martins \& J.A. Ventura, 2003. Índice de artrópodes pragas do mamoeiro (Carica papaya L.). Vitória, Incaper, $48 \mathrm{p}$.
Dantas, J.L.L., D.T. Junghans \& J.F. de Lima (Eds.), 2013. Mamão: o produtor pergunta, a Embrapa responde. Brasília: Embrapa Informação Tecnológica. Embrapa Mandioca e Fruticultura Tropical (Coleção 500 perguntas, 500 respostas), $170 \mathrm{p}$.

FAO (Food and Agriculture Organization of the United Nations), 2016. Disponível em: <http://www.fao.org >. [Acesso em: o8.viii.2018].

Faria, A.R.N., A.C. da S. Noronha, A.A.R. Oliveira, A.M.G. Oliveira, C.E.L. Cardoso, C.H.S.P. Ritzinger, E.J. de Oliveira, E.F. Coelho, H.P. Santos Filho, J.L. Cruz, J.R.P. Oliveira, J.L.L. Dantas, L.D. Souza, M. de A. Oliveira, M.A. Coelho Filho, N.F. Sanches, P.E. Meissner Filho, V.M. Medina \& Z.J.M. Coroleiro, 2009. A cultura do mamão. Brasília: Embrapa Informação Tecnológica. Embrapa Mandioca e Fruticultura Tropical (Coleção Plantar, 65). 119 p.

Feres, R.J.F., 200o. Levantamento de observações naturalísticas da acarofauna (Acari, Arachnida) de seringueiras cultivadas (Hevea spp., Euphorbiaceae) no Brasil. Revista Brasileira de Zoologia, 7: 157-173. DOI: https://doi.org/10.1590/s010181752000000100011.

Flechtmann, C.H.W. \& E.W. Baker, 1970. A preliminary report on the Tetranychidae (Acarina) of Brazil. Annals of the Entomological Society of America, 63: 156-163. DOI: https://doi.org/10.1093/aesa/63.1.156.

Flechtmann, C.H.W., 1967. Ácaros de plantas frutíferas. Piracicaba: USP (Boletim Técnico Científico, 30). 24 p.

Gallo, D., S. Silveira Neto, R.P.L. Carvalho, G.C. de Baptista, E. Berti Filho, J.R.P. Parra, R.A. Zucchi, S.B. Alves, J.D. Vendramim, L.C. Marchini, J.R.S. Lopes \& C. Omoto, 2002. Entomologia Agrícola. Piracicaba: FEALQ, 920 p.

Gioria, R., 2002. Doenças e pragas que atacam as orquídeas. Brasil Orquídeas, 64 p.

IBGE (Instituto Brasileiro de Geografia e Estatística), 2016. Produção agrícola municipal. Disponível em: <https://sidra.ibge.gov.br/tabela/5457\#resultado $>$. [Acesso em: 08.viii.2018].

Martins, D. dos S. \& S.L.D. Marin, 1998. Pragas do mamoeiro. In: Braga Sobrinho, L.B., J.E. Cardoso \& F. das Chagas (Eds.). Pragas de fruteiras tropicais de importância agroindustrial. Brasília: Embrapa Informação Tecnológica, 209 p. 
Mendonça, R.S., D. Navia, I.R. Diniz \& C.H.W. Flechtmann, 2011. South American spider mites: new hosts and localities. Journal of Insect Science, 11: 1-18. DOI: https://doi.org/10.1673/031.011.12101.

Moraes, G.J. de \& C.H.W. Flechtmann, 2008. Manual de acarologia. Acarologia básica e ácaros de plantas cultivadas no Brasil. Ribeirão Preto, Holos Editora, 308 p.

Moraes, G.J. de, 1981. Ácaros e insetos associados a algumas culturas irrigadas do Sub-médio São Francisco. Petrolina: Embrapa Semiárido. Embrapa Semiárido (Circular Técnica, 4), $32 \mathrm{p}$.

Nakayama, K., J.M. Abreu \& E.C.A. Ferraz, 1987. Controle Químico do ácaro Tetranychus mexicanus (McGregor, 1950) em mudas de cacaueiro. Revista Theobroma, 17: 9-16.

Oliveira, C.A.L. de, 1987. Ácaros, p. 104-110. In: Ruggiero, C. (Ed.). Cultura do maracujazeiro. Ribeirão Preto, Legis Summa, $250 \mathrm{p}$.

Paschoal A.D. \& P.R. Reis, 1968. Relação de ácaros encontrados em plantas. Revista de Agricultura, 43: 137-139.
Paschoal, A.D., 1968. Espécies de ácaros encontradas em plantas no Estado de São Paulo. Ciência e Cultura, 20: 258.

Paschoal, A.D., 1970. Revisão da família Tetranychidae no Brasil (Arachnida: Acarina). Anais da Escola Superior de Agricultura Luiz de Queiroz, 27: 457-483. DOI: https://doi.org/10.1590/s0071-12761970000100033.

Sousa, J.M. de, M.G.C. Gondim Júnior \& A.C. Lofego, 2010. Biologia de Tetranychus mexicanus (McGregor) (Acari: Tetranychidae) em três espécies de Annonaceae. Neotropical Entomogy, 39: 319-323. DOI: https://doi.org/10.1590/s1519$566 \times 2010000300002$.

Stein, C.P. \& N. Daólio, 2012. Biologia de Tetranychus mexicanus (McGregor) (Acari: Tetranychidae) em folhas de pupunha (Bactris gasipaes Kunth.). Bioikos, 26: 23-28.

\section{Suggestion citation:}

Santos, R.S., N.J. Ferla, J.J. Ferla \& W. Silva, 2018. Registro de Tetranychus mexicanus (McGregor) (Acari: Tetranychidae) em mamoeiro (Carica papaya L.) no estado do Acre, Brasil. EntomoBrasilis, 11 (2): 147-150.

Available on: doi:10.12741/ebrasilis.v11i2.764
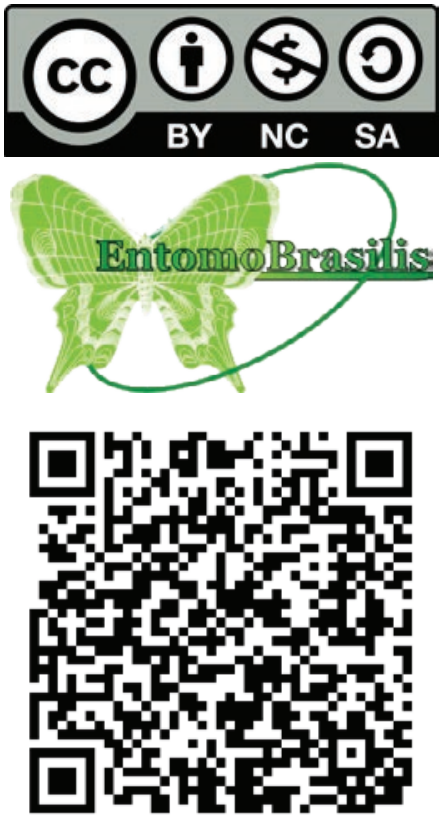